
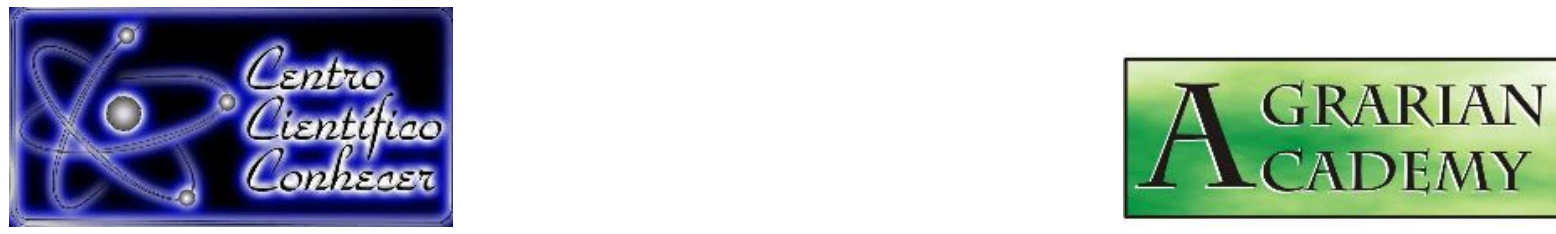

\title{
CLASSIFICAÇÃO DOS COEFICIENTES DE VARIAÇÃO PARA AS PROPRIEDADES DE PAINÉIS DE MADEIRA AGLOMERADA
}

Douglas Lamounier Faria1; Maria Cecíllia Ramos de Araújo Veloso; Thiago de Paula Protásio ${ }^{2}$; Lourival Marin Mendes ${ }^{1}$; José Benedito Guimarães Júnior ${ }^{1}$

1. Programa de Pós-Graduação em Engenharia de Biomateriais - UFLA 2. Professor Dr. do Curso de Engenharia Florestal - UFRA E-mail para correspondência: douglas.lamounier@yahoo.com

Recebido em: 02/06/2019 - Aprovado em: 15/06/2019 - Publicado em: 22/07/2019 DOI: 10.18677/Agrarian_Academy_2019a9

\begin{abstract}
RESUMO
Atualmente tem-se estudando bastante as propriedades de painéis produzidos com madeira reconstituída, em especial os aglomerados, principalmente se tratando de suas propriedades físico-mecânicas atribuídas. Entretanto, tais propriedades apresentam elevados índices de coeficiente de variação (CV), partindo de algumas classificações genéricas. Neste sentido, o objetivo desse trabalho foi gerar intervalos de classificação visando determinar a exatidão de experimentações nesta área. Para tanto, fez-se uso de CV das propriedades físico-mecânicas obtidos de artigos técnico-científicos relevantes sobre painéis de madeira reconstituída. Com base nos resultados gerou-se a seguinte classificação: Absorção de água: Baixo $=\mathrm{CV} \leq$ $7,22 \%$; médio = 7,22 < CV $\leq 27,78 \%$; alto $=27,78<\mathrm{CV} \leq 54,51 \%$ e muito alto $=\mathrm{CV}$ $>54,51 \%$. Para inchamento em espessura: Baixo $=\mathrm{CV} \leq 9,71 \%$; médio $=9,71<\mathrm{CV}$ $\leq 24,29 \%$; alto $=24,29<\mathrm{CV} \leq 39,12 \%$ e muito alto $=\mathrm{CV}>39,12 \%$. Para MOE: Baixo = CV $\leq 8,05 \%$; médio $=8,05<\mathrm{CV} \leq 20,98 \%$; alto $=20,98<\mathrm{CV} \leq 33,85 \%$ e muito alto $=\mathrm{CV}>33,85 \%$. Para MOR: Baixo $=\mathrm{CV} \leq 9,84 \%$; médio $=9,84<\mathrm{CV} \leq$ $24,83 \%$; alto $=24,83<\mathrm{CV} \leq 39,45 \%$ e muito alto $=\mathrm{CV}>39,45 \%$. Na tração perpendicular: Baixo $=\mathrm{CV} \leq 4,70 \%$; médio $=4,70<\mathrm{CV} \leq 33,88 \%$; alto $=33,98<\mathrm{CV}$ $\leq 48,88 \%$ e muito alto $=\mathrm{CV}>48,88 \%$.
\end{abstract}

PALAVRAS-CHAVE: Estabilidade dimensional; Precisão experimental. Variabilidade.

\section{CLASSIFICATION OF COEFFICIENT OF VARIATION THE PROPERTIES OF PARTICLEBOARD}

\begin{abstract}
At present, the properties of panels produced with reconstituted wood, especially the agglomerates, have been studied quite well, especially when dealing with their assigned physical-mechanical properties. However, these properties have high coefficient of variation (CV) indexes, starting from some generic classifications. In this sense, the objective of this work was to generate classification intervals in order to
\end{abstract}


determine the accuracy of experiments in this area. In order to do so, we used the $\mathrm{CV}$ of the physical-mechanical properties obtained from relevant technical-scientific articles on reconstituted wood panels. Based on the results the following classification was generated: Water absorption: Low $=\mathrm{CV} \leq 7.22 \%$; mean $=7.22$ $<\mathrm{CV} \leq 27.78 \%$; high $=27.78<\mathrm{CV} \leq 54.51 \%$ and very high $=\mathrm{CV}>54.51 \%$. For swelling in thickness: Low = CV $\leq 9.71 \%$; mean $=9.71<\mathrm{CV} \leq 24.29 \%$; high $=24.29$ $<\mathrm{CV} \leq 39.12 \%$ and very high $=\mathrm{CV}>39.12 \%$. For MOE: Low $=\mathrm{CV} \leq 8.05 \%$; mean $=$ $8.05<\mathrm{CV} \leq 20.98 \%$; high $=20.98<\mathrm{CV} \leq 33.85 \%$ and very high $=\mathrm{CV}>33.85 \%$. For MOR: Low = CV $\leq 9.84 \%$; mean = $9.84<\mathrm{CV} \leq 24.83 \%$; high $=24.83<\mathrm{CV} \leq 39.45 \%$ and very high $=\mathrm{CV}>39.45 \%$. In perpendicular traction: Low $=\mathrm{CV} \leq 4.70 \%$; mean $=$ $4.70<\mathrm{CV} \leq 33.88 \%$; high $=33.98<\mathrm{CV} \leq 48.88 \%$ and very high $=\mathrm{CV}>48.88 \%$.

KEYWORDS: Variability; Dimensional stability; Experimental precision.

\section{INTRODUÇÃO}

Os painéis produzidos com madeira reconstituída se originaram por causa da ausência da matéria-prima causada pela supressão de florestas nativas (FREIRE et al., 2015; SOZIM et al., 2019). O painel aglomerado começou a ser produzido no Brasil na década de 60, sendo formado por partículas de madeira e/ou de outros materiais lignocelulósicos com geometria reduzida aglutinadas com um adesivo sintético e consolidadas sob pressão e temperatura (PIERRE et al., 2014; CARVALHO et al., 2014; TRIANOSKI et al., 2015; BALDIN et al., 2016).

A aplicabilidade, destinação bem como classe de resistência de um painel de madeira estão diretamente relacionados com suas características intrínsecas, principalmente relacionados com sua estabilidade dimensional (capacidade de inchar quando da absorção de água), como também rigidez, resistência a carregamentos mecânicos. A qualidade dos painéis é norteada quanto a resistência e durabilidade nas propriedades de absorção de água, inchamento em espessura, tração perpendicular e flexão estática. Quando se faz a observação dessas características, muitos pesquisadores verificam altos valores de $\mathrm{CV}$, quando comparado com algumas classificações genéricas (MUZEL et al., 2015; GUIMARÃES JÚNIOR et al., 2016; ANDRADE et al., 2018; SHIROSAKI et al., 2019)

Triola (1999) define CV como a relação entre o desvio padrão e a média, sendo utilizado normalmente acerca da variação de variáveis assim como a exatidão de experimentos. Segundo Sampaio (1998), o CV é o parâmetro mais empregado para medir o quão instável encontra-se uma característica ou variável. Tem-se que para menores valores de CV, melhor será a homogeneidade dos dados e consequentemente reduzidas serão as variações do acaso.

Estudando valores de coeficientes de variação em ensaios agrícolas, Gomes (1985) fez sua classificação como mostrado a seguir: CV baixo quando se apresentaram inferiores a 10\%; CV médio entre 10 e 20\%; CV alto quando entre 20 e 30\%; CV muito alto quando acima de 30\%. Apesar de ser basear em dados agrícolas esta classificação serve como base para ser utilizada visando a classificação de inúmeras variáveis indiscriminadamente dentro da experimentação em geral, por exemplo painéis de madeira reconstituída.

Visando a determinação de limites na distribuição dos valores de CV, Garcia (1989) propôs utilizar a relação entre a média $(\bar{X})$ e o desvio padrão (s) dos valores de coeficiente de variação de diversos experimentos, envolvendo a mesma variável, da seguinte forma: Coeficiente de variação baixo: $\mathrm{CV} \leq \bar{X}-\mathrm{s}$, médio: $\bar{X}-\mathrm{s}<\mathrm{CV} \leq$ $\bar{X}+\mathrm{s}$, alto: $\mathrm{X}<\mathrm{CV} \leq \bar{X}+2 \mathrm{~s}$ e muito alto: $\mathrm{CV}>\bar{X}+2 \mathrm{~s}$. 
Porém, para ser válida esta classificação, os dados utilizados devem apresentar distribuição normal. Uma vez não apresentando esse comportamento, faz-se a utilização da metodologia estimulada pelo trabalho de Costa et al. (2002). Os autores sugeriram uma metodologia paralela de classificação dos valores de CV, podendo ser aplicada independentemente da distribuição de probabilidade dos valores de coeficiente de variação. Este método baseia-se no uso da mediana e do pseudo-sigma.

Desta maneira, este trabalho teve como objetivo apresentar uma possível classificação para os resultados encontrados para CV para as propriedades físicomecânicas para painéis aglomerados produzidos com madeira reconstituída.

\section{MATERIAL E MÉTODOS}

Para realização deste estudo, utilizou-se valores de CV de propriedades físicomecânicas de painéis aglomerados produzidos com madeira reconstituída partindo de trabalhos publicados em periódicos. Vale salientar que não foram especificados os delineamentos experimentais, utilizando o exposto por Estefanel et al. (1987), que afirmam que os mesmos não apresentariam diferenças nos valores de CV, tendo como pressuposição que a maneira como o experimento é estabelecido tem como objetivo remover a chance de possível erro experimental.

Os valores resultantes dos periódicos observados para CV foram tabelados para posteriormente realização de análise matemática. A primeira análise realizada foi a visualização da distribuição dos coeficientes de variação utilizando-se o histograma, acompanhado do teste de normalidade dos coeficientes de variação por meio do método Shapiro-Wilk, utilizando o programa computacional SISVAR. Foi considerado como critério de rejeição da hipótese $\mathrm{H}_{0}$ de normalidade a região crítica do teste tal que, $p(\mathrm{~W}<\mathrm{W})<0,05$; em que $\mathrm{w}$ é o valor da estatística do teste.

$O$ teste de normalidade foi utilizado para a seleção da metodologia a ser empregada na classificação do coeficiente de variação, ou seja, se os dados apresentarem distribuição normal pode-se utilizar a proposta feita por Garcia (1989), de forma direta. Para as variáveis que não apresentaram distribuição normal, utilizou-se a transformação logarítmica, obtendo faixas de classificação e, posteriormente, voltando os dados a escala original.

Para essas duas situações, as faixas de coeficiente de variação são assim classificadas: Baixo ( $\mathrm{CV} \leq \mathrm{m}-\mathrm{s})$; médio $(\mathrm{m}-\mathrm{s}<\mathrm{CV} \leq \mathrm{m}+\mathrm{s})$; alto $(\mathrm{m}+\mathrm{s}<\mathrm{CV} \leq \mathrm{m}+$ $2 \mathrm{~s})$; muito alto ( $\mathrm{CV}<\mathrm{m}+2 \mathrm{~s}$ ); sendo que "m" corresponde a média e "s" ao desvio padrão para os valores de coeficientes de variação.

Se a transformação logarítmica não conseguir promover a normalização dos dados, utiliza-se a metodologia proposta por Costa et al. (2002), onde as faixas de classificação de coeficientes de variação se fundamentam no uso da mediana (md) e pseudo-sigma (PS), os quais não necessitam ter distribuição normal dos dados.

Neste caso, a mediana dos coeficientes de variação $(\mathrm{md})$ é dada por $(\mathrm{Q} 1+$ Q3)/2, onde Q1 e Q3 são o primeiro e terceiro quartil respectivamente, os quais delimitam $25 \%$ de cada extremidade da distribuição dos coeficientes de variação e, o pseudo-sigma (DP) corresponde a IQR/1,35; sendo IQR representa a amplitude interquartílica (Q3 - Q1), que é uma medida resistente que indica o quanto os dados estão distanciados da mediana.

Assim, as faixas de coeficiente de variação ficam assim classificadas: Baixo $(\mathrm{CV} \leq \mathrm{md}-1 \mathrm{DP})$; médio (md - 1DP $<\mathrm{CV} \leq \mathrm{md}+1 \mathrm{DP})$; alto (md - 1DP $<\mathrm{CV} \leq \mathrm{md}+$ 2DP); e muito alto ( $C V>m d+2 D P)$. 


\section{RESULTADOS E DISCUSSÕES}

$\mathrm{Na}$ Tabela 1 estão apresentados os resultados obtidos para $\mathrm{W}$ e $\mathrm{pr}<\mathrm{W}$ utilizando o teste de Shapiro-Wilk para as propriedades físico-mecânicas analisadas.

TABELA 1. Resultados de W e pr<W para o teste de Shapiro-Wilk.

\begin{tabular}{lcc}
\hline Propriedade & \multicolumn{2}{c}{ Teste de Shapiro-Wilk } \\
\cline { 2 - 3 } & $\mathrm{W}$ & $\mathrm{pr}<\mathrm{W}$ \\
\hline Absorção de água & 0,9457 & $0,1292^{*}$ \\
Inchamento em & 0,9767 & $0,7149^{*}$ \\
espessura & & \\
MOE & 0,9720 & $0,3704^{*}$ \\
MOR & 0,9839 & $0,097^{*}$ \\
Tração perpendicular & 0,9394 & 0,0225 \\
\hline
\end{tabular}

*Indica presença de distribuição normal.

Após tratamento com logaritmo na base 10, observou-se na distribuição de frequências e no teste de normalidade que os CV's para as propriedades de absorção de água, inchamento em espessura, MOE e MOR apresentaram distribuição normal. Sendo assim, pode-se fazer o uso da metodologia para classificação de CV como mostrado por Garcia (1989).

Em relação à propriedade tração perpendicular, esta não apresentou distribuição normal, mesmo após tratamento estatístico. Com isso, fez-se o emprego da metodologia proposta por Costa et al. (2002). Situação esta que independe se as propriedades avaliadas apresentaram ou não distribuição normal. Observa-se na Tabela 2 os valores encontrados para as estatísticas descritivas referentes aos CV das propriedades físico-mecânicas dos painéis avaliados.

TABELA 2. Valores para estatísticas descritivas para as propriedades físicomecânicas dos painéis avaliados.

\begin{tabular}{cccc}
\hline Propriedade & Observações $(\mathrm{n})$ & Média (\%) & Desvio Padrão (\%) \\
\hline $\begin{array}{c}\text { Absorção de água } \\
\text { Inchamento em }\end{array}$ & 33 & 14,16 & 1,96 \\
espessura & 31 & 15,45 & 1,59 \\
MOE & 43 & 13,00 & 1,61 \\
MOR & 52 & 15,63 & 1,59 \\
Tração perpendicular & 44 & 19,91 & 1,75 \\
\hline
\end{tabular}

$\mathrm{Na}$ Tabela 2 observa-se maior número de CV para as propriedades MOR e tração perpendicular, apresentando médias para os respectivos valores de CV de 15,63 e 19,91\%. A propriedade física de absorção de água apresentou o maior desvio padrão entre todas propriedades físico-mecânicas avaliadas, com resultado de $1,96 \%$. Já o menor desvio padrão foi observado para MOR, com resultado de $1,59 \%$. São apresentados na Tabela 3 os resultados de CV para as propriedades físico-mecânicas dos painéis aglomerados avaliados. 
TABELA 3. Classificação dos coeficientes de variação para as propriedades físicomecânicas em painéis aglomerados.

\begin{tabular}{lcccc}
\hline \multirow{2}{*}{ Propriedade } & \multicolumn{3}{c}{ Classificação Coeficiente de Variação (\%) } \\
\cline { 2 - 5 } & Baixo & Médio & Alto & Muito alto \\
\hline $\begin{array}{l}\text { Absorção de água } \\
\text { Inchamento em }\end{array}$ & $\mathrm{CV} \leq 7,22$ & $7,22<\mathrm{CV} \leq 27,78$ & $27,78<\mathrm{CV} \leq 54,51$ & $\mathrm{CV}>54,51$ \\
espessura & $\mathrm{CV} \leq 9,71$ & $9,71<\mathrm{CV} \leq 24,29$ & $24,29<\mathrm{CV} \leq 39,12$ & $\mathrm{CV}>39,12$ \\
MOE & $\mathrm{CV} \leq 8,05$ & $8,05<\mathrm{CV} \leq 20,98$ & $20,98<\mathrm{CV} \leq 33,85$ & $\mathrm{CV}>33,85$ \\
MOR & $\mathrm{CV} \leq 9,84$ & $9,84<\mathrm{CV} \leq 24,83$ & $24,83<\mathrm{CV} \leq 39,45$ & $\mathrm{CV}>39,45$ \\
Tração & & & & \\
perpendicular & $\mathrm{CV} \leq 4,70$ & $4,70<\mathrm{CV} \leq 33,88$ & $33,88<\mathrm{CV} \leq 48,88$ & $\mathrm{CV}>48,88$ \\
\hline
\end{tabular}

Observa-se que cada variável apresentou faixa de valores dos coeficientes de variação específica, justificando ainda a necessidade de se considerar a natureza da variável na classificação dos coeficientes de variação. Visualiza-se, também, discordância entre esta classificação obtida e a proposta por Gomes (1985). Esta última é geralmente utilizada em trabalhos científicos apresentando limites fixos qualquer que seja a variável analisada.

De acordo com Gomes (1985) os coeficientes de variação são considerados baixos quando inferiores a $10 \%$. Neste trabalho observou-se que a todos os dados se mostraram abaixo dessa referência; quando a tração perpendicular apresentou o menor valor, com $4,7 \%$ e maior valor para MOR com $9,84 \%$.

Nos valores ditos médios, alto e muito alto; todos os testes apresentaram acima daqueles classificados por Gomes (1985); sendo que MOE foi a propriedade que mais se aproximou desta classificação, com valores de 8,05; 20,98 e 33,85\%; respectivamente. No limite superior (coeficiente de variação muito alto), observam-se os maiores valores para as propriedades de absorção de água, com $54,51 \%$, e tração perpendicular, com $48,80 \%$.

\section{CONCLUSÕES}

Com base nos resultados obtidos nesta pesquisa, gerou-se uma classificação para os coeficientes de variação para as propriedades dos painéis de madeira aglomerada:

$\checkmark$ Absorção de água: Baixo $=\mathrm{CV} \leq 7,22 \%$; médio $=7,22<\mathrm{CV} \leq 27,78 \%$; alto $=$ $27,78<\mathrm{CV} \leq 54,51 \%$ e muito alto $=\mathrm{CV}>54,51 \%$.

$\checkmark$ Para inchamento em espessura: Baixo $=\mathrm{CV} \leq 9,71 \%$; médio $=9,71<\mathrm{CV} \leq$ $24,29 \%$; alto $=24,29<\mathrm{CV} \leq 39,12 \%$ e muito alto $=\mathrm{CV}>39,12 \%$.

$\checkmark$ Para MOE: Baixo = CV $\leq 8,05 \%$; médio $=8,05<\mathrm{CV} \leq 20,98 \%$; alto $=20,98<$ $\mathrm{CV} \leq 33,85 \%$ e muito alto $=\mathrm{CV}>33,85 \%$.

$\checkmark$ Para MOR: Baixo $=\mathrm{CV} \leq 9,84 \%$; médio $=9,84<\mathrm{CV} \leq 24,83 \%$; alto $=24,83<$ CV $\leq 39,45 \%$ e muito alto $=\mathrm{CV}>39,45 \%$.

$\checkmark$ Para tração perpendicular: Baixo $=\mathrm{CV} \leq 4,70 \%$; médio $=4,70<\mathrm{CV} \leq$ $33,88 \%$; alto $=33,98<\mathrm{CV} \leq 48,88 \%$ e muito alto $=\mathrm{CV}>48,88 \%$.

\section{REFERÊNCIAS}

ANDRADE, L. M. F.; SCATOLINO, M. V.; FARIA, D. L.; CÉSAR, A. A.; MENDES, L. M.; GUIMARÃES JÚNIOR, J. B. Inclusão do resíduo de polpação da celulose na produção de painéis aglomerados de média densidade. Scientia Forestalis, v. 46, n. 120, 2018. 
BALDIN, T.; SILVEIRA, A. G.; VIDRANO, B. R. A.; CANCIAN, L. C.; SPATT, L. L.; HASELEIN, C. R. Qualidade de painéis aglomerados produzidos com diferentes proporções de madeira e capim-annoni. Revista Brasileira de Ciências Agrárias, v. 11 , n. 3, p. 230-237, 2016.

CARVALHO, A. G.; LELIS, R. C. C.; NASCIMENTO, A. M. Avaliação de adesivos à base de taninos de Pinus caribaea var. bahamensis e de Acacia mearnsii na fabricação de painéis aglomerados. Ciência Florestal, v. 24, n. 2, p. 479-489, 2014.

COSTA, N. H. A. D.; SERAPHIN, J. C.; ZIMMERMANN, F. J. P. Novo método de classificação de coeficientes de variação para a cultura do arroz de terras altas. Pesquisa Agropecuária Brasileira, v. 37, n. 3, p. 243-249, 2002.

ESTEFANEL, V.; PIGNATARO, I. A. B.; STORCK, L. Avaliação do Coeficiente de Variação de experimentos com algumas culturas agrícolas. In: Simpósio de Estatistica Aplicada à Experimentação Agronômica, 2., 1987, Londrina, Anais... Londrina: UEL, 1987. P. 115-131.

FREIRE, A. L. F.; FIGUEIRÊDO, M. C. B.; ROSA, M. F.; ARAÚJO JÚNIOR, C. P. Impactos ambientais de painéis de madeira e derivados - Uma revisão de literature. Espacios, v. 36, n. 10, p. 3-14, 2015.

GARCIA, C. H. Tabelas para classificação do Coeficiente de Variação. Piracicaba: IPEF, 1989, 12 p. (Circular Técnica, 171).

GOMES, F. Curso de estatística experimental. 11. ed. Piracicaba: ESALQ. 467 p, 1985.

GUIMARÃES JUNIOR, J. B.; XAVIER, M. M.; SANTOS, T. S.; PROTÁSIO, T. P.; MENDES, R. F.; MENDES, L. M. Inclusão de resíduo da cultura de sorgo em painéis aglomerados de eucalipto. Pesquisa Florestal Brasileira, v. 36, n. 88, p. 435- 442, 2016.

MUZEL, S. D.; GAVA, M.; CORTEZ-BARBOZA, J.; OLIVEIRA, K. A.; MORALES, E. A. M.; ARAÚJO, V. A. MDP panels manufactured with Hevea brasiliensis overlaid with bamboo foil of Phyllostachys edulis. Advanced in Materials Science, v. 1088, p. 686-689, 2015.

PIERRE, F. C.; BALLARIN, A. W.; PALMA, H. L. Caracterização física de painéis aglomerados de Eucalyptus grandis com adição de resíduos industriais madeireiros. Cerne, v. 20, n. 2, p. 321-328, 2014.

SAMPAIO, I. B. M. Estatística aplicada à experimentação animal. Belo Horizonte: Fundação de Ensino e Pesquisa em Medicina Veterinária e Zootecnia. 221 p, 1998.

SHIROSAKI, R. K.; ALMEIDA, T. H.; PANZERA, T. H.; CHRISTOFORO, A. L.; LAHR, F. A. R. Caracterização de painéis de partículas de média densidade feitos com resina poliuretana monocomponente à base de mamona. Construindo, v. 19, n. 1, p. 37-43, 2019. 
SOZIM, P. C. L.; NAPOLI, L. M.; FERRO, F. S.; MUSTEFAGA, E. C.; HILLIG, E. Propriedades de painéis aglomerados produzidos com madeiras de Ligustrum lucidum e Pinus taeda. Pesquisa florestal brasileira, v. 39, p. 1-8, 2019.

TRIANOSKI, R.; IWAKIRI, S.; NASCIMENTO, C. C.; BIL, N. F. Painéis aglomerados produzidos com quatro espécies de madeiras tropicais da Amazônia. Scientia Forestalis, v. 43, n. 106, p. 445-452, 2015.

TRIOLA, M. F. Introdução a estatística. LTC: 7 edição, 1999. 